\title{
Acupuncture Relieves Opioid-Induced Constipation in Clinical Cancer Therapy - A Meta-Analysis and Systematic Review
}

\author{
Changhui Han' \\ Yingjuan Liu $^{2}$ \\ Huanfang Fan' \\ Dehui $\mathrm{Li}^{1}$ \\ Na Guo'
}

'Affiliated Hospital of Hebei University of Traditional Chinese Medicine, Provincial Hospital of Traditional Chinese Medicine, Shijiazhuang, People's Republic of China; ${ }^{2}$ Institute of Cardiovascular Sciences, University of Manchester, Manchester, UK
Correspondence: Yingjuan Liu University of Manchester, 46 Grafton Street, Manchester, MI 3 9PT, UK Email liuyingjuan2008@gmail.com

\begin{abstract}
Cancer pain is a common problem in clinical cancer therapy. Opioid analgesia is one of the most effective drugs for pain relief with satisfying performance besides the side effect of opioid-induced constipation (OIC). Acupuncture, as a Chinese traditional noninvasive intervention, has been applied to clinical cancer pain management and functional constipation therapy. However, only a few studies have adopted this treatment for OIC patients. Due to limited numbers of investigated subjects and variability of application methods, including treatment apparatus, acupoints, durations, and sessions, the interpretation of acupuncture's therapy effects from single-site randomized clinical trials (RCT) is limited. Therefore, we conducted a meta-analysis by collecting published data from Pubmed, Embase, Cochrane library, and Web of Science. Five RCTs focusing on the application of acupuncture with or without medication in OIC patients were included. An overall remission rate of $86.8 \%$ in the acupuncture-treated group was achieved, higher than the control group (78.9\%; RR, 1.10, 95\% CI $[1.03,1.18])$. The symptom scores, reporting on defecation frequency, defecation straining, abdominal pain, defection time, and stool property, in acupuncture groups were lower than control groups with a standardized mean difference (SMD) of $-2.21[-4.15,-0.27]$. The quality of life (QOL) for patients in the acupuncture treated group increased compared to the control group with reduced PAC-QOL scores (SMD, $-1.02[-1.78,-0.26])$. Referring to the effects from pure acupuncture treatment (SMD, -0.43 $[-0.83,-0.03])$, the co-intervention of acupuncture and drugs (SMD, $-1.77[-2.51,-1.02])$ improved the life quality of patients more remarkably $(\mathrm{P}<0.05)$. Overall, our data confirmed the therapeutic effects of acupuncture in the treatment of OIC. The co-intervention of acupuncture with drugs improves the outcomes of OIC patients better than a single strategy. Combined therapy with both medicine and acupuncture has insightful potential for future clinical cancer patient management on constipation problems.
\end{abstract}

Keywords: acupuncture, opioid-induced constipation, cancer, pain, meta-analysis

\section{Introduction}

Opioid-induced constipation (OIC) is a common adverse effect of opioid therapy for cancer pain. About 30-70\% of cancer patients experience pain during all stages of the disease, such as anticancer treatment, curative treatment, and life-long treatment. ${ }^{1}$ Opioid analgesia is a frequently used medicine and is one of the most effective pain relief drugs. ${ }^{2}$ As a painkiller, opioid significantly improves the quality of life for cancer patients; however, the associated side effects discount its applicability. Especially constipation, the most common and long-lasting gastrointestinal complication of opioids, restricts opioid dosage for chronic pain 
control. $^{3,4}$ Among patients with opioid administration, $40 \%$ will develop constipation during their follow-up periods. $^{5}$

The strategies for OIC control are variable and case dependent. Reducing opioid dose, switching opioid subtypes, using laxatives, or adding $\mu$-opioid receptor antagonist, are frequently applied treatments in clinical practice. ${ }^{6}$ Acupuncture is a therapy method in Chinese traditional medicine. There are several types of acupuncture techniques, including electro-acupuncture, transcutaneous electrical acupoint stimulation (TEAS), and acupressure, are commonly used in clinical applications in addition to the traditional acupuncture. ${ }^{7}$ In contrast to conventional medication, acupuncture is suggested to be a safe and more effective treatment for functional constipation with fewer adverse events. ${ }^{8,9}$ Additionally, acupuncture has also been one of the most widely used non-invasive interventions for cancer-associated symptom management, particularly for cancer pain. ${ }^{10}$

The roles of acupuncture in both cancer pain and functional pain promote its application in OIC. Besides the recently published reports of clinical investigations, ${ }^{11,12}$ a few randomized clinical trials $(\mathrm{RCT})^{13-16}$ have been registered to evaluate the therapeutic potential of acupuncture in relieving OIC symptoms. There are systematic reviews that focus on the therapeutic effects of acupuncture on cancer pain ${ }^{10,17}$ or general constipation; ${ }^{18}$ however, no completed meta-analyses with specific interest on acupuncture and its application in OIC treatment are available. Since these RCT studies are typically single-centered and small-sized, the conclusion on the effectiveness of Acupuncture in OIC management is still questioned. Therefore, we conducted a systematic review and meta-analysis on the up-to-date RCT data of Acupuncture and OIC to address this question.

\section{Methods}

This review and meta-analysis were conducted compliant with PRISMA guideline. ${ }^{19}$ The protocol of this metaanalysis was described in Supplementary File 1.

\section{Search Strategy}

In August 2020, four databases, including Pubmed, Embase, Cochrane library, and Web of Science, were searched to identify the randomized clinical trial (RCT) studies reporting the application of Acupuncture on Opioid-induced constipation patients. The following items were used in the searches: (1) "opioid", or "opiate", or "opiate alkaloids", or "opioid analgesics", "Opioidinduced Constipation"; and (2) "constipation", or "Gastrointestinal Transit", or "slow transit", or "bowel dysfunction"; combined with (3) "acupuncture", or "Electro-acupuncture", or "transcutaneous electric stimulation" or "acupressure". No restriction was set for the study publication time or investigation regions. All studies with English abstracts were included and processed with the review protocol.

\section{Study Selection}

All studies identified from literature searches were reviewed in three stages, including title review, abstract review, and full-text review, by two reviewers $(\mathrm{CH}$ and YL) independently. Any discrepancy was solved by discussing and tracing back to the original studies according to the following inclusion and exclusion criteria.

\section{Inclusion Criteria}

1. Study design: randomized clinical trials with two or multiple groups regardless of the blinding to patients or assessors.

2. Study subjects: Patients 18 years old or over diagnosed with constipation due to opioids application for management of cancer pain or non-cancer associated pain.

3. Treatment methods: The intervention group received acupuncture (traditional acupuncture with needles), electro-acupuncture, transcutaneous electric stimulation, or acupressure, with or without the combination of the same medication as the control group. There is no limitation to the acupuncture types, acupoints, frequency, sessions, durations, and follow-up periods. There is no restriction to the treatment type of control groups who received no treatment, placebo, sham acupuncture, or conventional medication.

4. Outcome assessments: Clear criteria for therapy responses were defined. The number of patients who were cured or with effective relieves of symptoms and patients responding with limited or no effects were reported. Assessments of constipation symptoms on relevant aspects, including stool property, defecation frequency or duration time, defecation comfortable level or difficulty, and bowel movement, were reported. 


\section{Exclusion Criteria}

1. Patients received opioids and acupuncture for pain relief with the central outcome assessment of pain and other side effects. Any studies without precise diagnoses of constipation claimed before acupuncture treatments were excluded.

2. Studies on patients aged less than 18 years old with diagnoses of functional constipation were not included in the analysis.

3. Clinical trials without randomization, which may lead to obvious bias in the evaluation, were excluded.

4. Studies on specific populations, like pregnant women, colon cancer patients, and patients with other diseases (for example, irritable bowel syndrome) that influence bowel function directly or indirectly were excluded.

5. Study protocols of ongoing RCTs without completed data were excluded.

\section{Study Quality Assessment}

The quality of all the included studies was assessed with Cochrane risk of bias tool, ${ }^{20}$ on random sequence generation, allocation concealment, participants and personnel blinding, outcome assessment blinding, incomplete outcome data and exclusions, selective reporting, and other sources of bias. Each item was assessed as "high risk", "low risk", or "unclear". Two reviewers (CH and YL) conducted the quality check independently. Any discrepancy between the two reviewers was resolved by discussion.

\section{Data Extraction}

All included studies were extracted by two reviewers $(\mathrm{CH}$ and YL). Discrepancies in data extraction were resolved by tracking back to the original records and discussing between two reviewers. The standard data extraction Table 1 includes the following categories: study information (author, publication time, investigation time, population, and study design), participant data (patients diagnoses, control and intervention groups, number of patients, patient genders and ages), therapy methods (treatments for control group patients; acupuncture techniques, sessions, duration, frequency, and follow-up period, cointervention strategies with acupuncture in the intervention group), and outcomes (criteria and numbers of symptom remission, constipation symptom assessment, and life quality assessment). All relevant information regarding these data categories was collected for data analyses.

\section{Data Analyses}

The extracted data were analyzed using the $\mathrm{R}$ "metafor" package. Fixed effect models were applied based on a priori examination of $\mathrm{I}^{2}<50 \%$ in the heterogeneity test; otherwise, random effect models were used instead. The remission rate of acupuncture compared with control groups was extracted as dichotomous data and calculated by risk ratio (RR) and 95\% confidence interval (95\% CI). For continuous variables, such as life quality assessment scores, standardized mean differences (SMDs) were calculated before meta-analyses according to Murad's report on measures using different scales. ${ }^{21}$ The dataset for analyses (Supplementary Table 1) and different scales (Supplementary Tables 2-5) used by included studies were available in Supplementary File 2. The means with 95\% CI for SMDs were estimated for post-treatment data. Studies with different acupuncture techniques were analyzed together as the acupuncture group. Subgroup analyses were done to identify the potential factors influencing the heterogeneity among studies, including the co-intervention strategies. The significance level was defined as $\mathrm{P}<0.05$.

\section{Results \\ Study Characteristics}

From 99 unique records identified from literature searches, five studies ${ }^{11,22-25}$ (a total of 656 participants) were included in this meta-analysis following the reviewing process shown in the PRISMA flow chart (Figure 1). All included studies were hospital-based and single-centered randomized clinical trials (RCTs). Figure 2 shows that the overall risk of bias of included studies is low or unclear. All studies are randomized for group allocations and nonselective in outcome reporting. The high-risk bias comes from participant and personal blinding procedures, where the blinding is not feasible due to the acupuncture approaches. The reporting biases from allocation concealment, outcome assessment blinding, and other nonspecified biases are unclear.

\section{Remission Rate}

Table 1 shows the reported study designs (sample sizes, therapy method, acupuncture points, and follow-up periods) and the number of cured or remised OIC patients following acupuncture or control therapies for all included studies. The acupuncture is mainly delivered through electrical methods, including interferential 


\begin{tabular}{|c|c|c|c|c|c|c|}
\hline \multicolumn{2}{|c|}{ 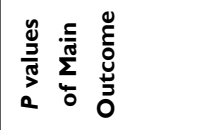 } & ๙్సి & $\begin{array}{l}\stackrel{n}{0} \\
\stackrel{i}{i}\end{array}$ & $\begin{array}{l}\text { 菅 } \\
\text { in }\end{array}$ & $\begin{array}{l}n \\
\stackrel{\Delta}{\mathrm{v}}\end{array}$ & $\frac{m}{0}$ \\
\hline \multirow{2}{*}{ 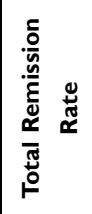 } & 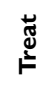 & 令 & ô & $\stackrel{\infty}{\infty}$ & o̊ & S. \\
\hline & 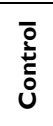 & 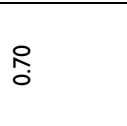 & $\stackrel{\substack{\infty \\
\hdashline}}{\circ}$ & $\stackrel{\infty}{0}$ & i̊ & $\stackrel{\infty}{\infty}$ \\
\hline \multirow{2}{*}{ 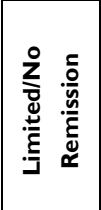 } & 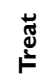 & $\approx$ & - & n & $N$ & 으 \\
\hline & $\begin{array}{l}\overline{0} \\
\overline{0} \\
\dot{0} \\
0\end{array}$ & 이 & $\sigma$ & $\alpha$ & $\alpha$ & $\underline{\infty}$ \\
\hline \multirow{2}{*}{ 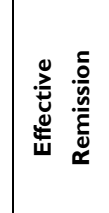 } & 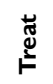 & ケ & $\hat{\lambda}$ & $\underline{m}$ & 으 & ธิ \\
\hline & ờ & $\mathscr{f}$ & $\stackrel{\sim}{2}$ & $\simeq$ & $\underline{m}$ & $\pi$ \\
\hline \multirow{2}{*}{ 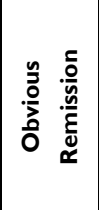 } & 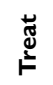 & 이 & $m$ & $\underline{\infty}$ & \pm & q \\
\hline & 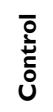 & $\bar{N}$ & N & $\underline{\text { 음 }}$ & n & $\stackrel{\infty}{\sim}$ \\
\hline \multirow{2}{*}{ ठั } & 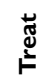 & & N & N & t & $\simeq$ \\
\hline & 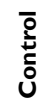 & & - & 0 & - & $\underline{m}$ \\
\hline \multicolumn{2}{|c|}{ 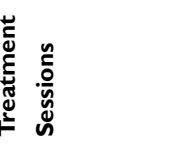 } & 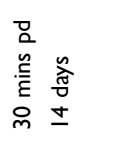 & 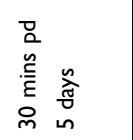 & 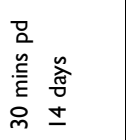 & 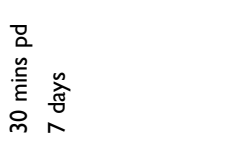 & 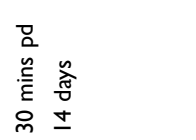 \\
\hline \multicolumn{2}{|c|}{ 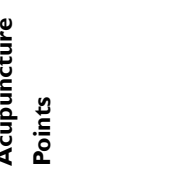 } & 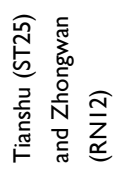 & 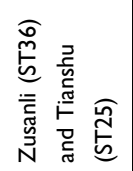 & 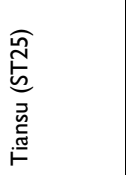 & 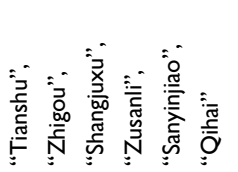 & 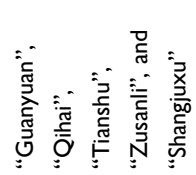 \\
\hline \multirow{2}{*}{ 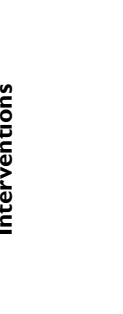 } & 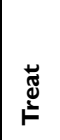 & 岁 & 凹 & 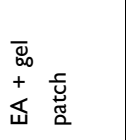 & 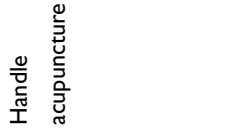 & 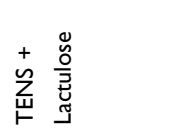 \\
\hline & ن̀ & 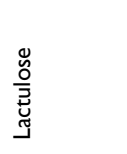 & 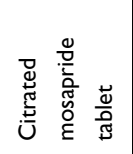 & 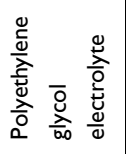 & 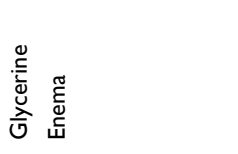 & 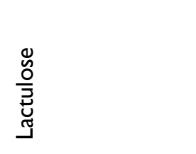 \\
\hline \multirow{2}{*}{ 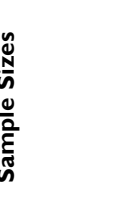 } & 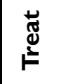 & $\stackrel{\infty}{\circ}$ & $m$ & g & 요 & $\stackrel{ \pm}{\simeq}$ \\
\hline & 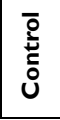 & 음 & $\stackrel{m}{m}$ & $\bar{\sigma}$ & 요 & $\hat{\underline{\Xi}}$ \\
\hline \multicolumn{2}{|c|}{ 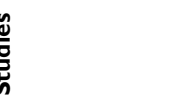 } & 蛋 & 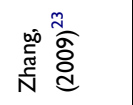 & 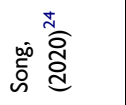 & 望 & 灾 \\
\hline
\end{tabular}




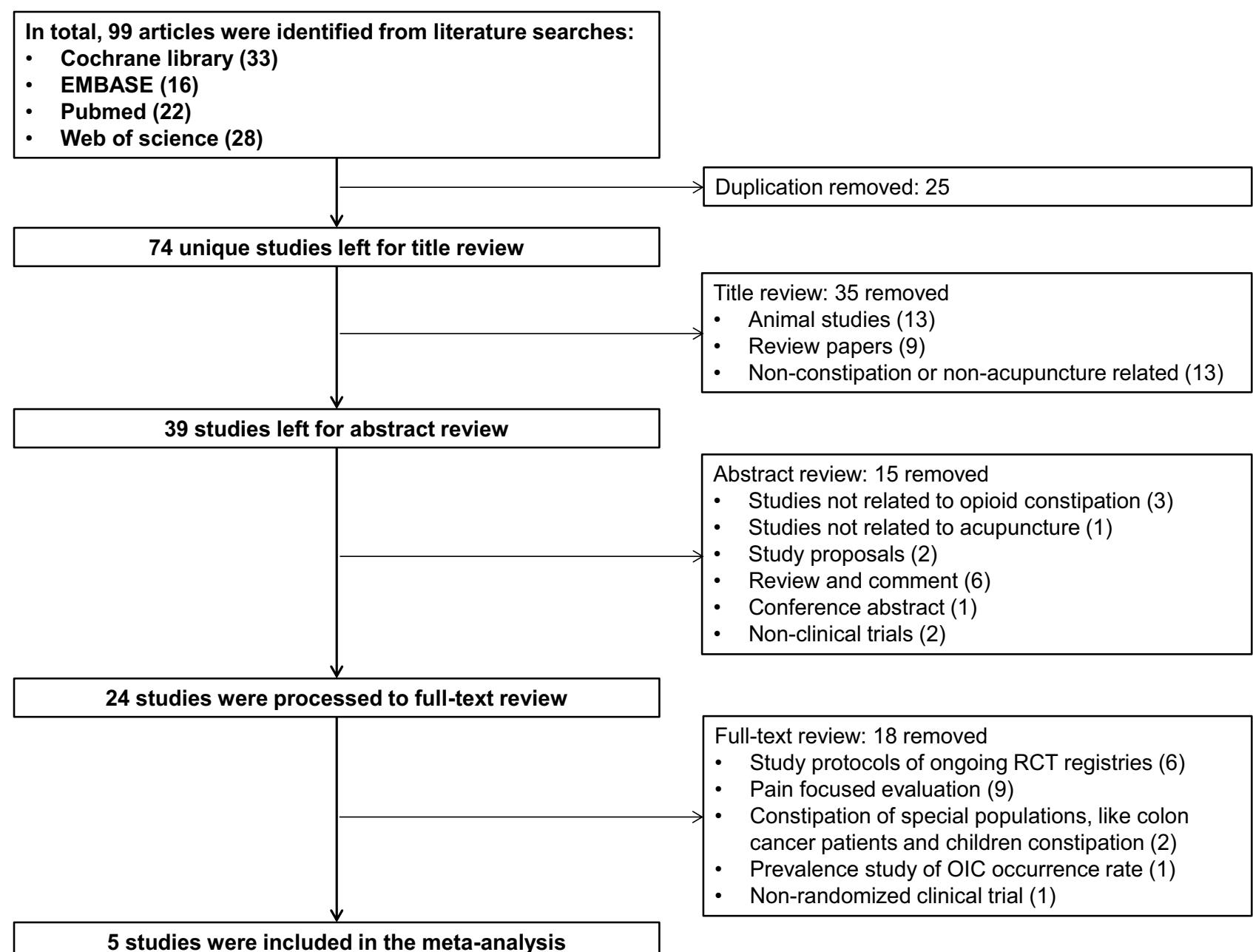

Figure I PRISMA diagram of the review. The diagram describes the procedures conducted for meta-analyses, including literature searches, review processes, records excluded in each process, and the final studies included for data extraction and analyses.

current (IFC) stimulation, transcutaneous electrical nerve stimulation (TENS), and electro-acupuncture (EA); while only Jiang's paper $^{25}$ in 2010 applied traditional Acupuncture for OIC patients. Two studies conducted acupuncture along with co-interventions. Cai's paper ${ }^{11}$ performed the acupuncture along with oral lactulose, the medication same as its control group, while Song's paper $^{24}$ carried out the acupuncture with a gel pad containing Chinese herb. The other three studies treated the patients with mere acupuncture in treatment groups and drugs in control groups. The number of acupoints in included studies ranges from one to six, with Tianshu (ST25) being applied in all five studies. Other commonly used acupoints are Zusanli (ST36), Qihai (CV6 or Ren 6), and Shangjuxu (ST37). The treatment durations for acupuncture are 30 minutes per session each day with 5-14 days.
All included studies reported the cure or remission rates following the Ministry of Health of Peoples Republic of China Guidelines for clinical research of new Chinese medicines, Applied Research on Traditional Chinese Medicine Spleen and Stomach Theory, or Criteria of clinical disease diagnoses and therapeutic effects judgment. The guidelines are of similar criteria in defining cases as cured or remised Supplementary Table 2 in Supplementary File 2). Cured patients are those with symptoms, including defection time, stool properties, and bowel movements that disappeared or recovered to presickness conditions. Patients with obvious remission are those with markedly relieving symptoms. Effective cases are those with symptoms improved and shorted defecation intervals by 24 hours.

Figure $3 \mathrm{~A}$ shows the overall remission rate (including cured, obvious remission, and effective remission) is 


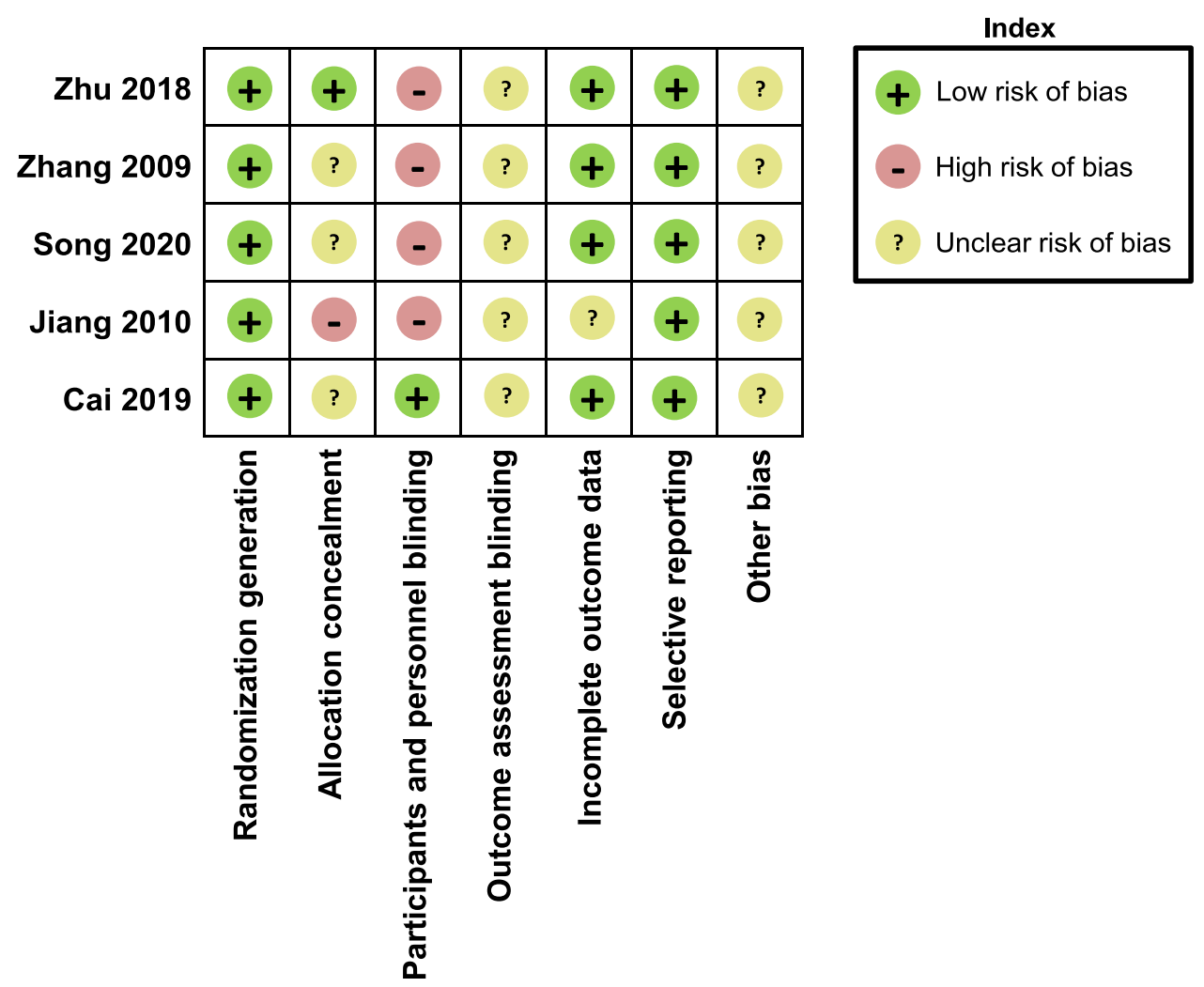

Figure 2 Risk of bias assessment for included studies. The evaluation of all included studies on their reporting biases according to the Cochrane risk of bias tool.

higher in the acupuncture-treated group than the control group (86.8\% vs 78.9\%; RR: 1.10 [1.03, 1.18]). Except for Zhu's paper ${ }^{22}$ in 2018 , all other four studies reported the cured rate of acupuncture treatment (Figure 3B) ranging from $5.0 \%$ to $13.3 \%$ (Median, $7.8 \%$ ) comparing with the control group with a range from $0 \%$ to $10.2 \%$ (Median, $3.2 \%$ ) without statistical significance (RR: 1.36 [0.72, 2.57]). The obvious remission rates (Figure 3C) following acupuncture therapies (32.3\% [range: 9.1-46.7\%]) are significantly higher than control group $(22.0 \%$ [range: $6.1-$ $36.6 \%]$ ) with an RR of 1.47 [1.14, 1.89]; while the rates of effective remission (Figure 3D) are of no significant difference between acupuncture group and control group (RR: $0.91[0.78,1.05])$. The statistical test shows the homogeneity among all five included studies $\left(\mathrm{I}^{2}=0 \%\right.$, $\mathrm{P}=0.61$ ). The funnel plot (Figure 4 ) is visually symmetric, indicating the absence of publication bias (Eggers' test: coefficient $=1.25, \mathrm{P}=0.254$ ).

\section{Symptom Scores}

All five studies evaluated the constipation symptoms but following different scales. Defecation frequency, defecation straining/difficulty, abdominal pain, defection time, and stool property are the standard items in applied symptom scoring scales. The comparison of items in each scale and their scoring criteria are available in Supplementary Table 3 of Supplementary File 2. For all scales used in included studies, higher symptom scores represent more severe constipation symptoms.

To synthesis the data, the overall symptom scores were transformed into standardized mean differences (SMDs). Meta-analysis using the random-effect model in Figure 5A indicates significantly lower symptom scores in the acupuncture group than the control group (SMD: -2.21 $[-4.15,-0.27])$. The reduction of symptom scores suggested better symptom-relieving effects of acupuncture treatment in comparison to control drug therapy. Significant heterogeneity was observed among included studies $\left(\mathrm{I}^{2}=64 \%, \mathrm{P}=0.03\right)$. The contour-enhanced funnel plot and Eggers' test did not indicate the presence of publication bias (coefficient $=2.01, \mathrm{P}=0.15$ ).

Subgroup analyses in Figure 5B were produced according to co-interventional strategies (drug administrations same as the control group) accompanying acupuncture. In studies without co-interventions, acupuncture therapy showed no apparent advanced effects in relieving 


\section{A Remission or cured in overall}

\begin{tabular}{|c|c|c|c|c|c|c|}
\hline Study & $\begin{array}{l}\text { Experim } \\
\text { Events }\end{array}$ & $\begin{array}{l}\text { ental } \\
\text { Total }\end{array}$ & $\begin{array}{r}\mathrm{Cc} \\
\text { Events }\end{array}$ & $\begin{array}{l}\text { ontrol } \\
\text { Total }\end{array}$ & Weight & $\begin{array}{c}\text { Risk ratio } \\
\text { MH, Fixed, 95\% Cl }\end{array}$ \\
\hline Zhu, H. D. 2018 & 75 & 98 & 70 & 100 & $26.8 \%$ & $1.09[0.92 ; 1.29]$ \\
\hline Zhang, F. L. 2009 & 32 & 33 & 29 & 33 & $11.2 \%$ & $1.10[0.96 ; 1.27]$ \\
\hline Song, N. 2020 & 33 & 40 & 32 & 41 & $12.2 \%$ & $1.06[0.85 ; 1.31]$ \\
\hline Jiang, Y. L. 2010 & 28 & 30 & 21 & 30 & $8.1 \%$ & $1.33[1.04 ; 1.72]$ \\
\hline Cai, H. 2019 & 114 & 124 & 109 & 127 & $41.6 \%$ & $1.07[0.98 ; 1.17]$ \\
\hline $\begin{array}{l}\text { Total }(95 \% \mathrm{Cl}) \\
\text { Prediction interva } \\
\text { Heterogeneity } \text { Tau }^{2}\end{array}$ & $=0.003$ & 325 & & 331 & $100.0 \%$ & $\begin{array}{c}1.10[1.03 ; 1.18] \\
{[0.90 ; 1.35]}\end{array}$ \\
\hline
\end{tabular}

\section{B Cured}

\begin{tabular}{|c|c|c|c|c|c|c|}
\hline Study & $\begin{array}{l}\text { Experim } \\
\text { Events }\end{array}$ & $\begin{array}{l}\text { ental } \\
\text { Total }\end{array}$ & $\begin{array}{r}\text { C } \\
\text { Events }\end{array}$ & $\begin{array}{r}\text { ontrol } \\
\text { Total }\end{array}$ & Weight & $\begin{array}{c}\text { Risk ratio } \\
\text { MH, Fixed, } 95 \% \mathrm{Cl}\end{array}$ \\
\hline Zhu, H. D. 2018 & & 98 & & 100 & $0.0 \%$ & \\
\hline Zhang, F. L. 2009 & 2 & 33 & 1 & 33 & $6.7 \%$ & $2.00[0.19 ; \quad 21.00]$ \\
\hline Song, N. 2020 & 2 & 40 & 0 & 41 & $0.7 \%$ & $21.52[0.04 ; 12064.52]$ \\
\hline Jiang, Y. L. 2010 & 4 & 30 & 1 & 30 & $6.7 \%$ & $4.00[0.47 ; \quad 33.73]$ \\
\hline Cai, H. 2019 & 12 & 124 & 13 & 127 & $86.0 \%$ & $0.95[0.45$ \\
\hline $\begin{array}{l}\text { Total }(95 \% \mathrm{Cl}) \\
\text { Prediction interval }\end{array}$ & & 325 & & 331 & $100.0 \%$ & $\begin{array}{c}1.36[0.72 ; 2.57] \\
{[0.05 ; 52.93]}\end{array}$ \\
\hline
\end{tabular}

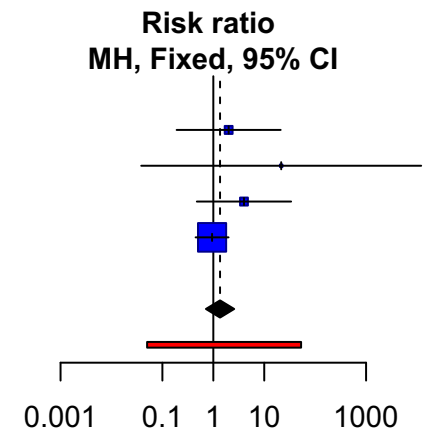

\section{Obvious remission}

\begin{tabular}{|c|c|c|c|c|c|c|}
\hline Study & $\begin{array}{l}\text { Experim } \\
\text { Events }\end{array}$ & $\begin{array}{l}\text { iental } \\
\text { Total }\end{array}$ & $\begin{array}{r}\mathrm{Cc} \\
\text { Events }\end{array}$ & $\begin{array}{l}\text { ontrol } \\
\text { Total }\end{array}$ & Weight & $\begin{array}{c}\text { Risk ratio } \\
\text { MH, Fixed, } 95 \% \mathrm{Cl}\end{array}$ \\
\hline Zhu, H. D. 2018 & 30 & 98 & 21 & 100 & $28.8 \%$ & $1.46[0.90 ; 2.36]$ \\
\hline Zhang, F. L. 2009 & 3 & 33 & 2 & 33 & $2.8 \%$ & $1.50[0.27 ; 8.40]$ \\
\hline Song, N. 2020 & 18 & 40 & 15 & 41 & $20.5 \%$ & $1.23[0.72 ; 2.09]$ \\
\hline Jiang, Y. L. 2010 & 14 & 30 & 7 & 30 & $9.7 \%$ & $2.00[0.94 ; 4.25]$ \\
\hline Cai, H. 2019 & 40 & 124 & 28 & 127 & $38.3 \%$ & $1.46[0.97 ; 2.22]$ \\
\hline $\begin{array}{l}\text { Total }(95 \% \mathrm{Cl}) \\
\text { Prediction interva } \\
\text { Heterogeneity: Tau }{ }^{2}\end{array}$ & $=0005$ & 325 & 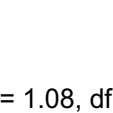 & 331 & $100.0 \%$ & $\begin{aligned} & 1.47[1.14 ; 1.89] \\
& {[1.06 ; 2.00] } \\
= & 0 \%\end{aligned}$ \\
\hline
\end{tabular}

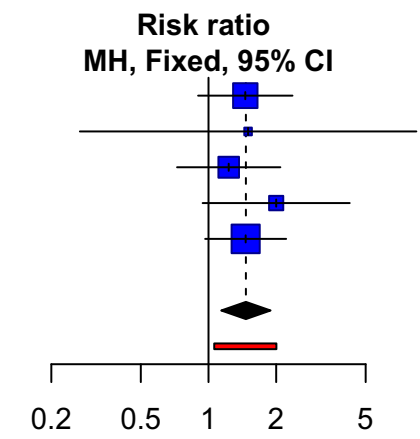

\section{Effective remission}

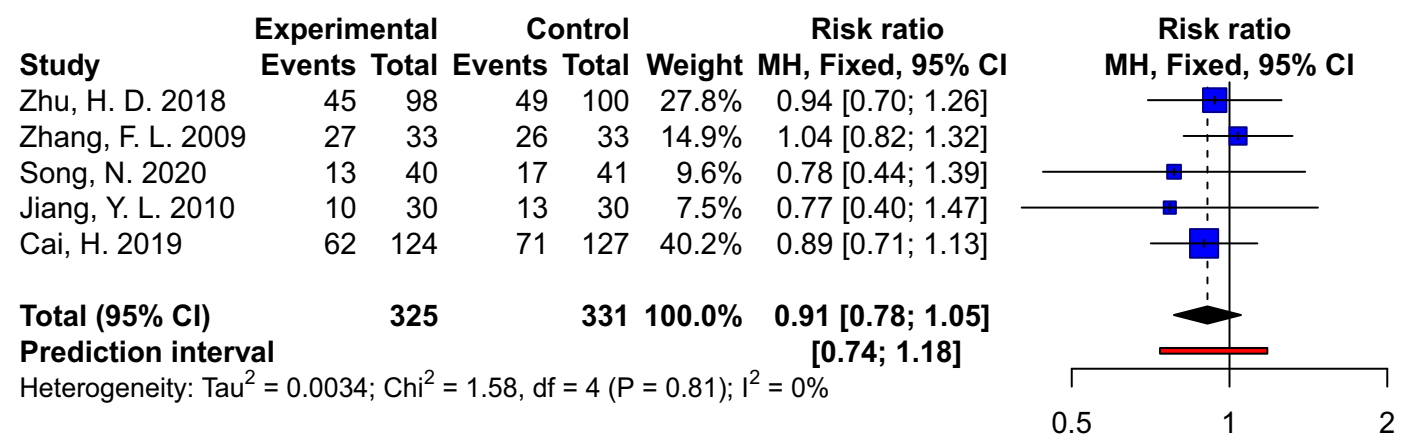

Figure 3 Remission rates of constipation symptoms in OIC patients. RRs were calculated to estimate the therapy outcomes of acupuncture in comparison with Control groups. (A) Overall remission rate including cured cases, obvious remissions, and effective remissions; (B) cured rate; (C) rate of obvious remissions; (D) rate of effective remissions. 


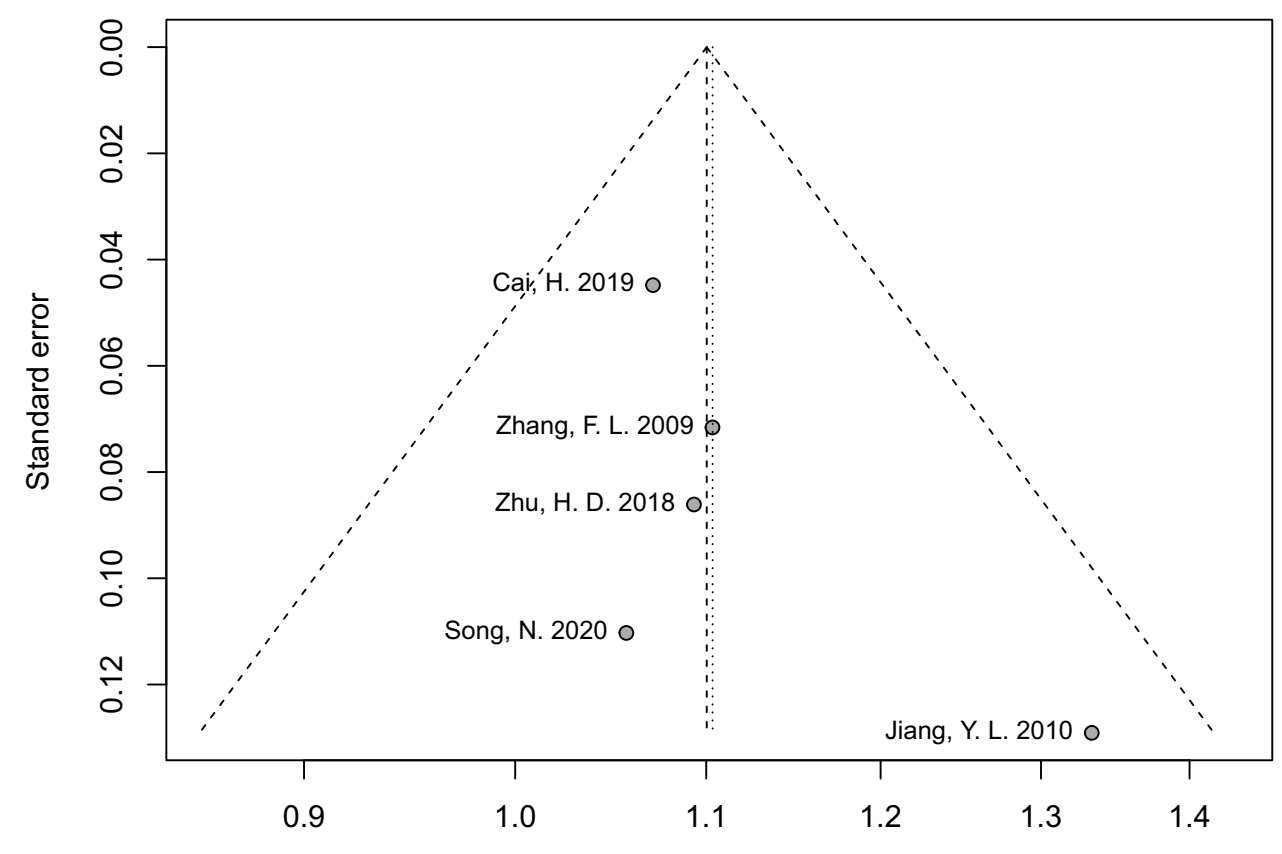

Hedges' g

Figure 4 The funnel plot of cured or remission rate data. Grey rectangles represent included studies.

\section{A Symptom score (overall)}

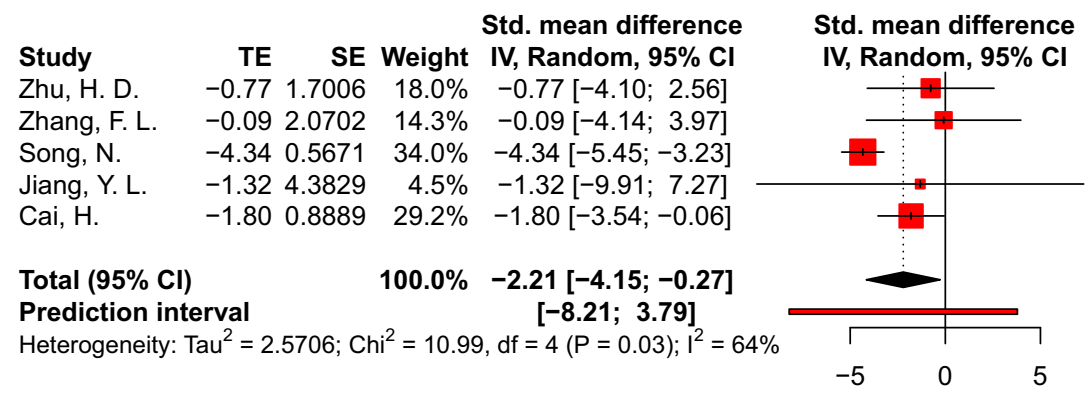

\section{B Symptom score (subgroup)}

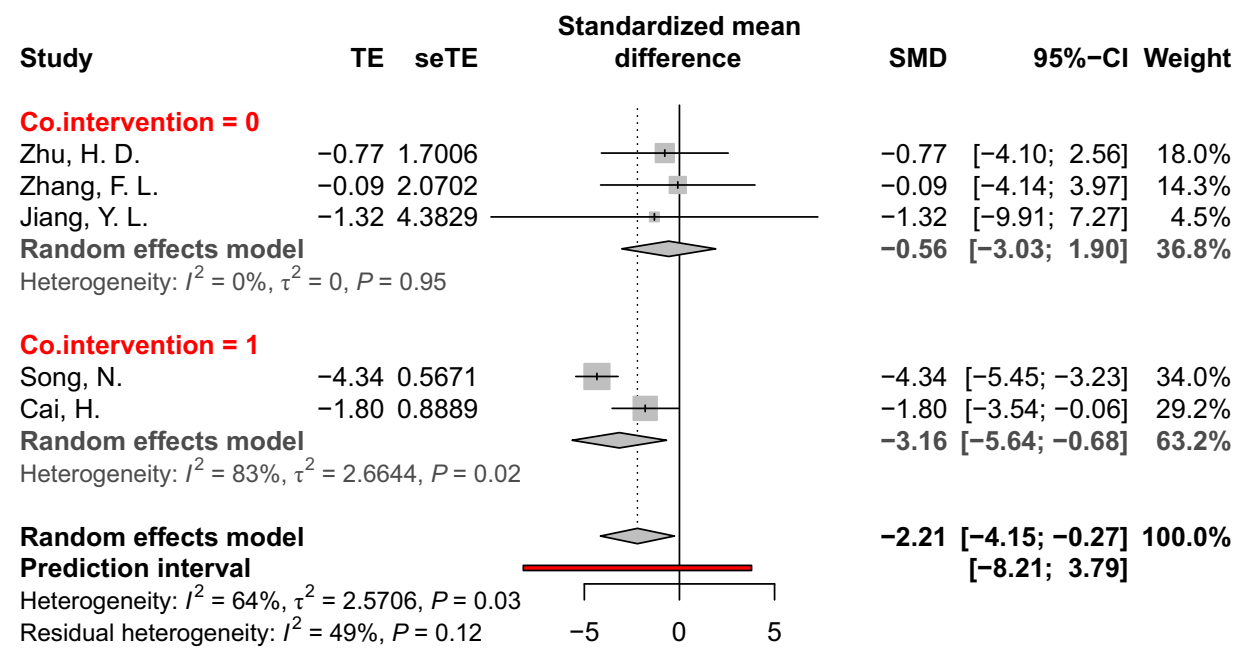

Figure 5 Meta-analyses of symptom scores and subgroup analyses for studies with or without co-intervention. (A) The overall symptom scores calculated with standardized mean difference referring to the control group. (B) The subgroup analyses of symptom scores defining by acupuncture with or without other co-intervention strategies. 


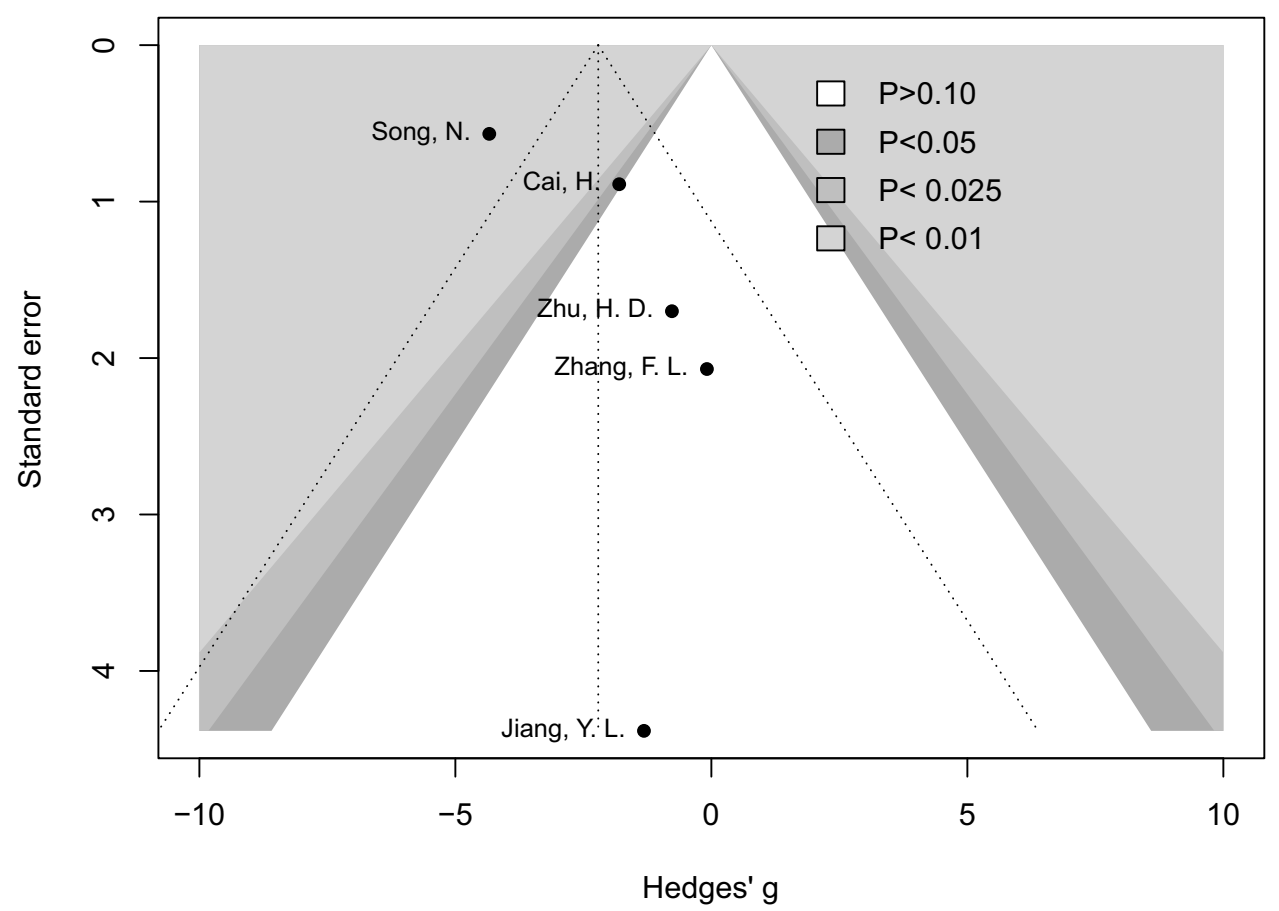

Figure 6 Contour-enhanced funnel plot of acupuncture versus control for symptom scores. Black dots represent included studies. Gradient grey colors indicate different significant levels.

constipation symptoms than control groups (SMD: -0.56 $[-3.03,1.90])$. Simultaneously, there was no significant heterogeneity identified among these three studies $\left(\mathrm{I}^{2}=0 \%, \mathrm{P}=0.95\right)$. The summarised data from studies using combined acupuncture with co-interventions indicated a substantial reduction of symptom scores in the acupuncture group than the control group (SMD: -3.16 $[-5.64,-0.68])$. However, heterogeneity was still examined between the two studies with accompanying drug applications with acupuncture therapy $\left(\mathrm{I}^{2}=83 \%, \mathrm{P}=0.02\right)$. This suggests the factor of co-intervention partially explained the resource of heterogeneity. Contourenhanced funnel plot (Figure 6) was symmetric, the Eggers' test of which is non-significant (coefficient= $2.014, \mathrm{P}=0.15$ ), suggesting no obvious publication bias of included studies.

\section{Life Qualities}

Four of the included studies reported the quality of life as an indicator for therapeutic effect evaluation. Except Jiang's study ${ }^{25}$ using Karnofsky Performance Status Scale (KPS, sample copy in Supplementary Table 4 of Supplementary File 2), the other three studies investigated the life qualities of OIC patients at pre- and post-treatment stages using the Patient Assessment of Constipation
Quality of Life Questionnaire (PAC-QOL) ${ }^{26}$ (sample copy in Supplementary Table 5 of Supplementary File 2). $\mathrm{KPS}^{27}$ is $0-100 \%$, stating the functionality and performance of targets, whereby the $100 \%$ means normal conditions without diseases, while lower percentages mean severe sickness. ${ }^{28}$ PAC-QOL is developed as 28 items reporting physical discomfort, psychosocial discomfort, worries and concerns, and satisfaction, with a scoring range from 0 to 4 points for each item. ${ }^{26,29}$ Higher scores indicate worse quality of life.

In combining the data from different scales, all scores were transformed into averaged points per item at $0-4$ scale according to PAC-QOL. KPS scores were inverted by subtracting from 100 and divided by 25 to fit into the 0-4 range of PAC-QOL. Due to the variability of scales, SMDs for each study were calculated for the acupuncturetreated group against the control group. Figure 7A shows that the QOL scores in the acupuncture-treated group are lower than the control group with statistical significance (SMD: $-1.02[-1.78,-0.26])$. There was significant heterogeneity among studies $\left(\mathrm{I}^{2}=74 \%, \mathrm{P}<0.01\right)$. Contourenhanced funnel plot (Figure 8) was visibly asymmetric; however, Eggers' test is not significant (coefficient= $-4.65, \mathrm{P}=0.08$ ), which suggested there was no apparent presence of publication bias of the included studies. 


\section{A Quality of life (overall)}

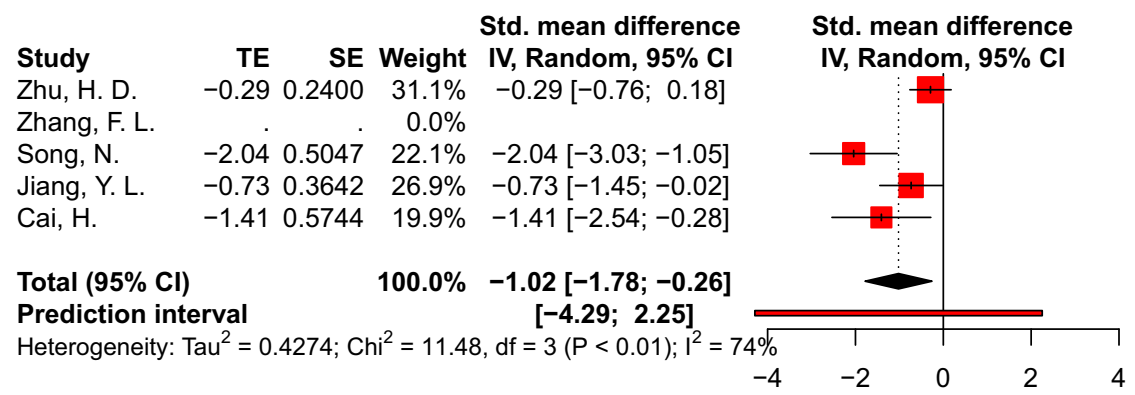

\section{B Quality of life (subgroup)}

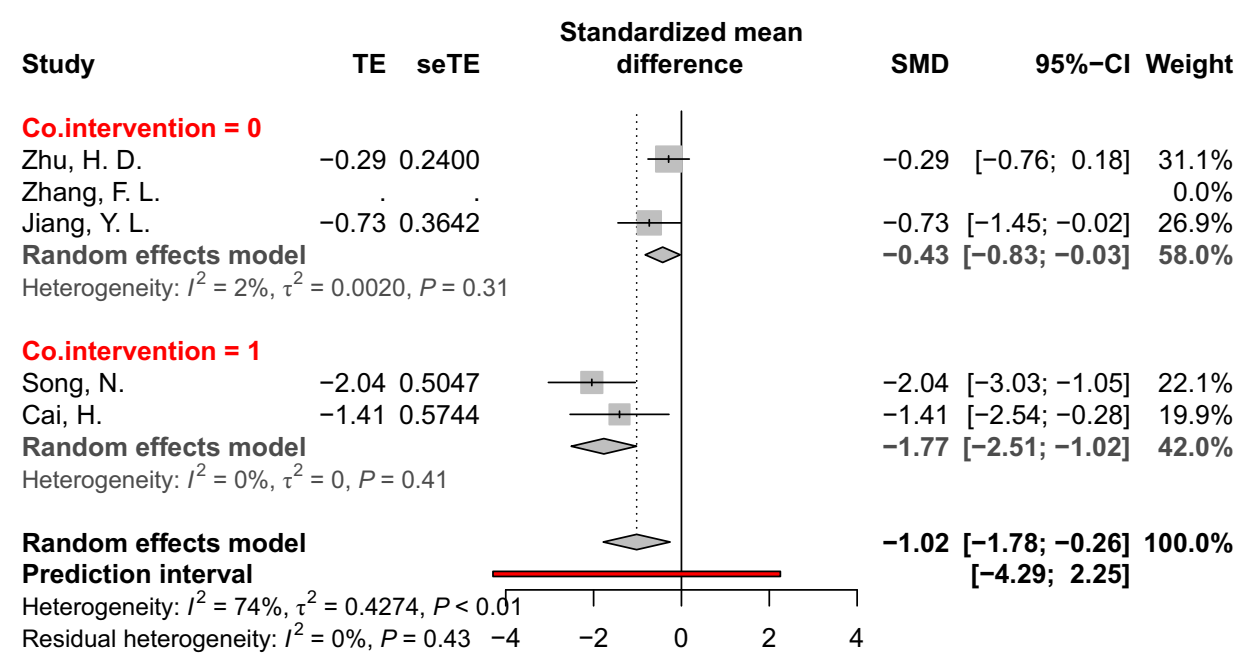

Figure 7 Meta-analyses of quality of life scores and subgroup analyses for studies with or without co-intervention strategies. (A) The overall quality of life scores calculated with standardized mean difference comparing to the control group. (B) The subgroup analyses of the quality of life scores defining by acupuncture with or without cointerventions. No quality of life score was reported in Zhang, F.L.'s paper; therefore, a $0.00 \%$ weight was allocated in the analyses.

Following acupuncture with or without co-intervention strategies, quality of life SMDs were processed for subgroup analyses. No obvious heterogeneity was identified in both subgroups (studies without co-intervention: $\mathrm{I}^{2}=2 \%$, $\mathrm{P}=0.31$; studies with co-intervention: $\mathrm{I}^{2}=0 \%, \mathrm{P}=0.41$ ). This suggested that co-interventions for acupuncture were the main source of heterogeneity between all included studies. The overall SMD for studies without co-intervention was $-0.43[-0.83,-0.03]$, while the summarised SMD for studies with co-intervention was $-1.77[-2.51,-1.02]$. These data suggested that the effects of acupuncture alone promoted the quality of life of OIC patients moderately; however, the combined therapeutic effects of acupuncture and drugs were remarkable in improving the quality of life for patients.

\section{Discussion}

The present meta-analyses of RCT trials indicated an improved therapeutic effect of acupuncture in clinical
OIC patient management. Even though acupuncture alone did not show a higher rate of curation, the obvious remission rate was significantly increased in comparison to control groups with the administration of drugs, such as lactulose, one of the commonly used stimulant laxatives for constipation. ${ }^{30}$ The outcomes of remission rates were evaluated at the end of continuous acupuncture therapy sessions after one or two weeks, which is the commonly used time durations for outcome evaluations in acupuncture treatment of other diseases. ${ }^{31,32}$ However, the effective onset times of laxatives in inducing defecations are suggested to be $6-72$ hours. ${ }^{33}$ Drugs are supposed to be rapid in relieving constipation, while acupuncture is more effective in the long-term management of OIC. The identified advantages of acupuncture over medications in this study are consistent with Wang's study on chronic functional constipation, which indicated the long-lasting effects of acupuncture for 5-12 weeks. $^{34}$ 


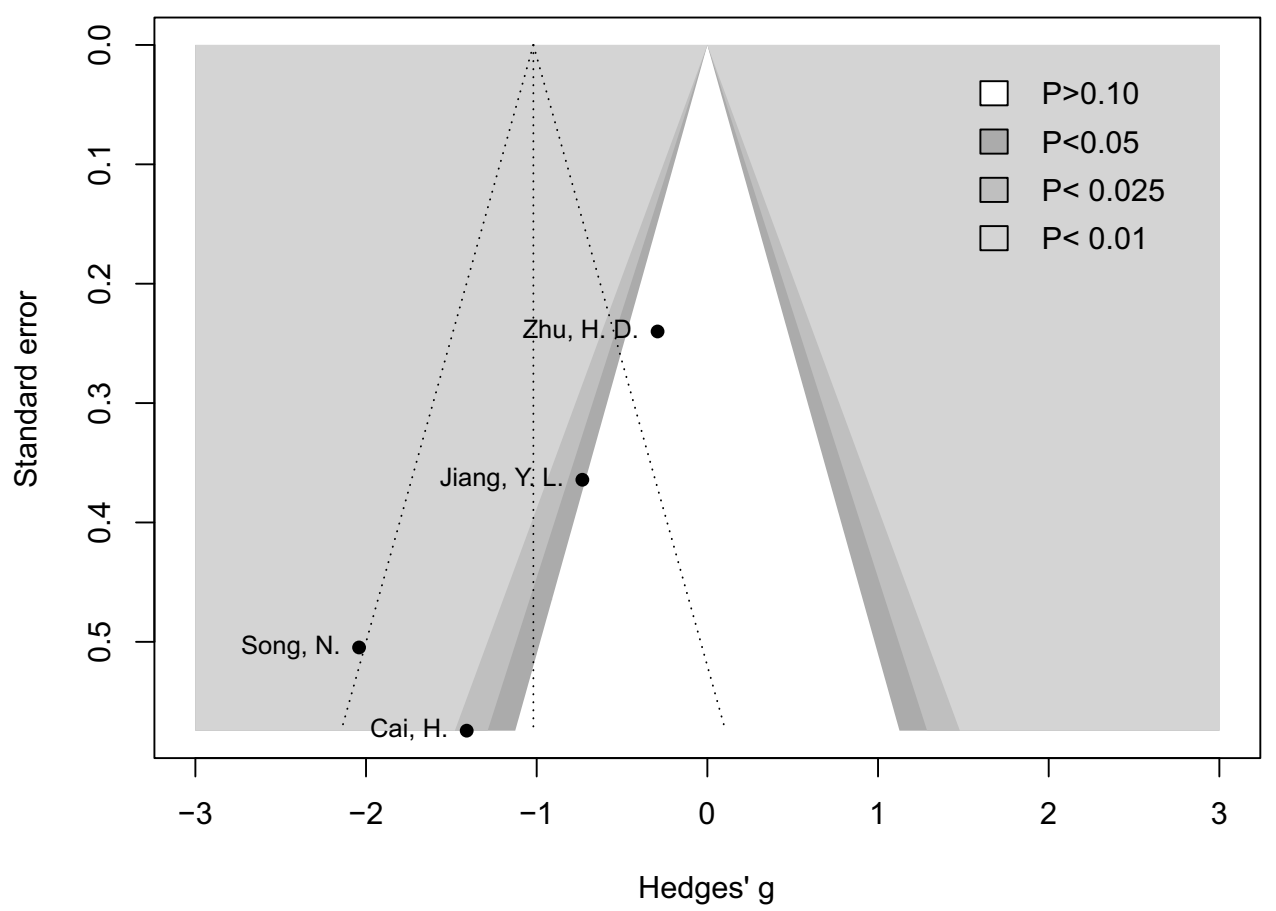

Figure 8 Contour-enhanced funnel plot of acupuncture versus control for quality of life scores. Black dots represent included studies. Gradient grey colors indicate different significant levels.

The overall symptom scores of constipation were reduced upon acupuncture treatment, especially when acupuncture was applied in combination with medications. The single intervention of acupuncture was adequate; however, it is not superior in relieving symptoms of OIC in contrast to general drug therapies. Apart from laxatives, many kinds of drugs, such as stool softeners, and prokinetic agents, are also commonly recommended for constipation treatment; however, side effects like flatus, headache, nausea, and diarrhea are reported. ${ }^{35}$ In contrast, the side effects of acupuncture are rarely reported. ${ }^{36}$ The most frequently observed ones are tiny hemorrhage and hematoma at the acupoints. ${ }^{37}$ Considering the minor side effects of acupuncture compared with drug prescriptions, acupuncture is suggested to be a safer option to choose for the clinical management of OIC patients. The combination of acupuncture and medication promotes the relieving effects of symptoms than mere acupuncture or drug administration, indicating a synergistic effect between acupuncture and medicine in the therapy of OIC. This phenomenon agrees with the enhancer role of acupuncture for drugs observed in treating other diseases, like Parkinson's disease $^{38}$ and methamphetamine addiction. ${ }^{39}$

In addition to the moderate improvement of therapeutic effects of acupuncture versus drug applications, the quality of life of OIC patients has significantly improved regardless of the mere usage of acupuncture or with co-interventional prescriptions. Besides constipation, acupunctures are a widely applied method for relieving cancer-related pains. ${ }^{10,40}$ A common associated symptom of constipation or gastrointestinal disorders is abdomen pain. ${ }^{41}$ Simultaneously, OICs are due to the clinical usage of opioids, one of the most widely used pain-relieving drugs in cancer patients. ${ }^{42}$ The pain-controlling effects of acupunctures prompt the therapy outcomes of OIC and cancer pains by increasing patients' quality of life remarkably than pure medication.

\section{Limitations}

There are limited studies available for a better interpretation of the therapy effects on OIC patients with different acupuncture techniques. In this study, all relevant papers, regardless of the applied acupuncture methods, including electro-acupuncture, transcutaneous electrical nerve stimulation, and traditional acupuncture, are analyzed together. Acknowledging the reported similar efficiency of transcutaneous electrical nerve stimulation and traditional acupuncture in treating chronic back pain, ${ }^{43}$ the varied acupuncture ways and acupoints will not cause apparent biases to the conclusion of this meta-analysis. However, the 
heterogeneity effects of different acupuncture techniques need to be considered in actual clinical applications. The guidelines or evaluation scales applied in included studies are not the same. With SMD transformation, the difference evaluations from these scales have been reduced. To improve the confidence of the results, additional studies in the future need to be included for updated analyses.

\section{Conclusions}

Acupuncture is an effective therapeutic strategy for the clinical management of opioid-induced constipation and enhances the effects of commonly prescribed drugs. The improvement of life qualities for OIC patients is more prominent with the pain-relieving potential for cancer patients. Acupuncture is an appropriate supportive method to be applied additionally to drug administrations for cancer patients with OIC symptoms.

\section{Abbreviations}

OIC, opioid-induced constipation; RCT, randomized clinical trial; QOL, quality of life; SMD, standardized mean difference; PAC-QOL, Patient Assessment of Constipation Quality of Life Questionnaire; KPS, Karnofsky Performance Status Scale.

\section{Copyright and Licenses}

Under the license agreement with Mapi Research Trust (MRT), the sample copy of PAC-QOL is granted to be reproduced in publications. PAC-QOL (Supplementary Table 5 in Supplementary File 2) contact information and permission to use: Mapi Research Trust, Lyon, France, https://eprovide.mapi-trust.org.

\section{Data Sharing Statement}

The dataset supporting the conclusions of this article, scales for symptom scoring system, and quality of life scales are included in the article as Supplementary Tables 1-5 in Supplementary File 2.

\section{Author Contributions}

All authors contributed to data analysis, drafting, or revising the article, have agreed on the journal to which the article will be submitted, gave final approval of the version to be published, and agree to be accountable for all aspects of the work.

\section{Funding}

This work was supported by the Scientific Research Project of Hebei Administration of Traditional Chinese
Medicine (Grants No. 2019030). The funding body played no role in the design of the study and collection, analysis, and interpretation of data and in writing the manuscript.

\section{Disclosure}

The authors declare that they have no competing financial interests.

\section{References}

1. Fallon M, Giusti R, Aielli F, et al. Management of cancer pain in adult patients: ESMO clinical practice guidelines. Ann Oncol. 2018;29:iv166-iv191. doi:10.1093/annonc/mdy152

2. Rosenblum A, Marsch LA, Joseph H, Portenoy RK. Opioids and the treatment of chronic pain: controversies, current status, and future directions. Exp Clin Psychopharmacol. 2008;16(5):405-416. doi:10.1037/a0013628

3. McNicol E, Horowicz-Mehler N, Fisk RA, et al. Management of opioid side effects in cancer-related and chronic non-cancer pain: a systematic review. J Pain. 2003;4(5):231-256. doi:10.1016/ S1526-5900(03)00556-X

4. Bell TJ, Panchal SJ, Miaskowski C, Bolge SC, Milanova T, Williamson R. The prevalence, severity, and impact of opioid-induced bowel dysfunction: results of a US and European Patient Survey (PROBE 1). Pain Med. 2009;10(1):35-42. doi:10.1111/j.1526-4637.2008.00495.x

5. Prichard D, Norton C, Bharucha AE. Management of opioid-induced constipation. $B r \quad J$ Nurs. 2016;25(10):S4-S11. doi:10.12968/ bjon.2016.25.10.S4

6. Neefjes ECW, van der Wijngaart $\mathrm{H}$, van der Vorst MJ, et al. Optimal treatment of opioid induced constipation in daily clinical practice an observational study. BMC Palliat Care. 2019;18(1):31. doi:10.1186/s12904-019-0416-7

7. Lu W, Rosenthal DS. Acupuncture for cancer pain and related symptoms. Curr Pain Headache Rep. 2013;17(3):321. doi:10.1007/ s11916-013-0321-3

8. Zhang T, Chon TY, Liu B, et al. Efficacy of acupuncture for chronic constipation: a systematic review. Am J Chin Med. 2013;41 (04):717-742. doi:10.1142/S0192415X13500493

9. Tao B, Chao S, Cuihong Z, Guangying H. Acupuncture for the treatment of functional constipation. J Trad Chin Med. 2016;36 (5):578-587. doi:10.1016/S0254-6272(16)30076-0

10. Hu C, Zhang H, Wu W, et al. Acupuncture for pain management in cancer: a systematic review and meta-analysis. Evid Based Complement Alternat Med. 2016;2016:1720239. doi:10.1155/2016/ 1720239

11. Cai H, Zhou Q, Bao G, Kong X, Gong LY. Transcutaneous electrical nerve stimulation of acupuncture points enhances therapeutic effects of oral lactulose solution on opioid-induced constipation. $J$ Int Med Res. 2019;47(12):6337-6348. doi:10.1177/0300060519874539

12. Wang PM, Hsu CW, Liu CT, Lai TY, Tzeng FL, Huang CF. Effect of acupressure on constipation in patients with advanced cancer. Support Care Cancer. 2019;27(9):3473-3478. doi:10.1007/s00520019-4655-1

13. Nederlands Trial Register. Effect of electroacupuncture on opioid-induced constipation in patients with cancer. ClinicalTrials. gov. Identifier: NCT03797586; 2019. Available from: https://clinical trials.gov/ct2/show/NCT03797586. Accessed May 12, 2021.

14. Nederlands Trial Register. The clinical efficacy of acupuncture as an adjunct to methadone treatment services for heroin addicts. ClinicalTrials.gov. Identifier: NCT01512433; 2012. Available from: https:/clinicaltrials.gov/ct2/show/NCT01512433. Accessed May 12, 2021. 
15. Nederlands Trial Register. Evaluating transcutaneous electrical nerve stimulation for postoperative pain after video-assisted thoracotomy surgery. ClinicalTrials.gov. Identifier: NCT01046695; 2010. Available from: https://clinicaltrials.gov/show/NCT01046695. Accessed May 12, 2021.

16. Australian New Zealand Clinical Trials Registry. Effect of electro-acupuncture on opioid consumption by patients with chronic pain. Registration number: ACTRN12609000676213; 2009. Available from: https://www.anzctr.org.au/Trial/Registration/ TrialReview.aspx?ID=308008. Accessed May 12, 2021.

17. He Y, Guo X, May BH, et al. Clinical evidence for association of acupuncture and acupressure with improved cancer pain: a systematic review and meta-analysis. JAMA Oncol. 2019;6(2):271-278. doi:10.1001/jamaoncol.2019.5233

18. Wang L, Xu M, Zheng Q, Zhang W, Li Y. The effectiveness of acupuncture in management of functional constipation: a systematic review and meta-analysis. Evid Based Complement Alternat Med. 2020;2020:6137450.

19. Liberati A, Altman DG, Tetzlaff J, et al. The PRISMA statement for reporting systematic reviews and meta-analyses of studies that evaluate healthcare interventions: explanation and elaboration. BMJ. 2009;339:e1-e34.

20. Higgins JPT, Altman DG, Gøtzsche PC, et al. The Cochrane collaboration's tool for assessing risk of bias in randomized trials. $B M J$. 2011;343:d5928. doi:10.1136/bmj.d5928

21. Murad MH, Wang Z, Chu H, Lin L. When continuous outcomes are measured using different scales: guide for meta-analysis and interpretation. BMJ. 2019;364:k4817. doi:10.1136/bmj.k4817

22. Zhu HD, Gong Z, Hu BW, Wei QL, Kong J, Peng CB. The efficacy and safety of transcutaneous acupoint interferential current stimulation for cancer pain patients with opioid-induced constipation: a prospective randomized controlled study. Integr Cancer Ther. 2018;17(2):437-443. doi:10.1177/1534735417734910

23. Zhang FL, Lin HS, He QY. Effect of electro-acupuncture in treating morphine sulfate caused constipation in tumor patients. Chin J Integr Med. 2009;29(10):922-925.

24. Song N, Meng LZ, Cheng J, et al. Ultrasonic Chinese herbal medication penetration therapy at Tianshu (ST25) for opioid-induced constipation with liver stagnation and spleen deficiency. World $J$ Acupunct Moxibustion. 2020;30(1):13-18. doi:10.1016/j.wjam.2020.01.002

25. Jiang YL, Wang QM. Observation of therapeutic effects of acupuncture therapy on treating constipation caused by opium analgesic treating tumor in 30 cases. Chin J Trad Med Sci Technol. 2010;17 (6):544-545.

26. Marquis P, De La Loge C, Dubois D, McDermott A, Chassany O. Development and validation of the patient assessment of constipation quality of life questionnaire. Scand $J$ Gastroenterol. 2005;40 (5):540-551. doi:10.1080/00365520510012208

27. Mor V, Laliberte L, Morris JN, Wiemann M. The Karnofsky performance status scale: an examination of its reliability and validity in a research setting. Cancer. 1984;53(9):2002-2007. doi:10.1002/10970142(19840501)53:9<2002::AID-CNCR2820530933>3.0.CO;2-W

28. Péus D, Newcomb N, Hofer S. Appraisal of the Karnofsky performance status and proposal of a simple algorithmic system for its evaluation. BMC Med Inform Decis Mak. 2013;13(1):72. doi:10.1186/1472-6947-13-72

Clinical Epidemiology

\section{Publish your work in this journal}

Clinical Epidemiology is an international, peer-reviewed, open access, online journal focusing on disease and drug epidemiology, identification of risk factors and screening procedures to develop optimal preventative initiatives and programs. Specific topics include: diagnosis, prognosis, treatment, screening, prevention, risk factor modification,

Submit your manuscript here: https://www.dovepress.com/clinical-epidemiology-journal
29. Nikjooy AP, Jafari HP, Saba MAMP, Ebrahimi NMP, Mirzaei RMD Patient assessment of constipation quality of life questionnaire: translation, cultural adaptation, reliability, and validity of the Persian version. Iran J Med Sci. 2018;43(3):261-268.

30. Daniali M, Nikfar S, Abdollahi M. An overview of interventions for constipation in adults. Expert Rev Gastroenterol Hepatol. 2020;14 (8):721-732. doi:10.1080/17474124.2020.1781617

31. Chen YJ, Chen CT, Liu JY, Shimizu Bassi G, Yang YQ. What is the appropriate acupuncture treatment schedule for chronic pain? Review and analysis of randomized controlled trials. Evid Based Complement Alternat Med. 2019;2019:5281039.

32. Shi Y, Zheng H, Zhou S, et al. Efficacy and safety of acupuncture for patients with chronic urticaria: study protocol of a randomized, sham-controlled pilot trial. Trials. 2019;20(1):326. doi:10.1186/ s13063-019-3433-1

33. Portalatin M, Winstead N. Medical management of constipation. Clin Colon Rectal Surg. 2012;25(1):12-19. doi:10.1055/s-0032-1301754

34. Wang Y, Liu Y, Zhou K, et al. The duration of acupuncture effects and its associated factors in chronic severe functional constipation: secondary analysis of a randomized controlled trial. Therap Adv Gastroenterol. 2019;12:1756284819881859. doi:10.1177/1756284819881859

35. Liu LW. Chronic constipation: current treatment options. Can J Gastroenterol. 2011;25(Suppl B):22B-28B. doi:10.1155/2011/360463

36. Ceniceros S, Brown GR. Acupuncture: a review of its history, theories, and indications. South Med J. 1998;91(12):1121-1125. doi:10.1097/00007611-199812000-00005

37. Ernst G, Strzyz H, Hagmeister H. Incidence of adverse effects during acupuncture therapy - a multicentre survey. Complement Ther Med. 2003;11(2):93-97. doi:10.1016/S0965-2299(03)00004-9

38. Hwang T-Y, Song M-A, Ahn S, et al. Effects of combined treatment with acupuncture and Chunggan formula in a mouse model of Parkinson's disease. Evid Based Complement Alternat Med. 2019;2019:3612587. doi:10.1155/2019/3612587

39. Hyo Lee B. A synergistic effect of combination of acupuncture and herb on the methamphetamine's reinforcement. $J$ Acupunct Meridian Stud. 2020;13(2):71. doi:10.1016/j.jams.2020.03.012

40. Chiu HY, Hsieh YJ, Tsai PS. Systematic review and meta-analysis of acupuncture to reduce cancer-related pain. Eur $J$ Cancer Care. 2017;26(2):e12457. doi:10.1111/ecc.12457

41. Bharucha AE, Chakraborty S, Sletten CD. Common functional gastroenterological disorders associated with abdominal pain. Mayo Clinic Proc. 2016;91(8):1118-1132. doi:10.1016/j.mayocp.2016.06.003

42. Gatti A, Sabato AF. Management of opioid-induced constipation in cancer patients. Clin Drug Investig. 2012;32(5):293-301. doi:10.2165/11598000-000000000-00000

43. Itoh $\mathrm{K}$, Itoh $\mathrm{S}$, Katsumi $\mathrm{Y}$, Kitakoji $\mathrm{H}$. A pilot study on using acupuncture and transcutaneous electrical nerve stimulation to treat chronic non-specific low back pain. Complement Ther Clin Pract. 2009;15(1):22-25. doi:10.1016/j.ctcp.2008.09.003 systematic reviews, risk \& safety of medical interventions, epidemiology \& biostatistical methods, and evaluation of guidelines, translational medicine, health policies \& economic evaluations. The manuscript management system is completely online and includes a very quick and fair peer-review system, which is all easy to use. 